\title{
Sex differences in striatal dopamine: in vivo microdialysis and behavioral studies
}

\author{
Stacy A. Castner ${ }^{\mathrm{a}}$, Li Xiao ${ }^{\mathrm{a}}$ and Jill B. Becker ${ }^{\mathrm{a}, \mathrm{b}}$ \\ a Psychology Department, Reproductive Sciences Program and ${ }^{b}$ Neuroscience Program, The University of Michigan, Ann Arbor, \\ MI 48104-1687 (USA)
}

(Accepted 1 December 1993)

Key words: Amphetamine; Estrogen; Ovariectomy; Castration; Dopamine release; Stereotyped behavior; Quantitative microdialysis

\begin{abstract}
Experiments were conducted to examine sex differences in striatal dopamine function using in vivo microdialysis in freely moving rats. We report here a sex difference in basal extracellular striatal dopamine determined by quantitative microdialysis (the no net flux method) when castrated and ovariectomized rats were compared. There was no sex difference in dopamine uptake into synaptosomes. This indicates that the sex difference in extracellular dopamine is most likely due to sex differences in dopamine release, synthesis, and/or metabolism. Within 30 min after a single injection (s.c.) of either estradiol benzoate $(2.0 \mu \mathrm{g} / 100 \mathrm{~g})$ or $17 \beta$-estradiol $(1.5 \mu \mathrm{g} / 100 \mathrm{~g})$ the amphetamine-stimulated release of dopamine was enhanced in the striatum of ovariectomized rats, but there was no effect in castrated male rats. The enhanced amphetamine-induced striatal dopamine release in ovariectomized rats was associated with an enhanced frequency of stereotyped head and limb movements and an increased peak in extra $1 / 4$ turns. There were also sex differences in stereotyped behavior and extra $1 / 4$ turns whether or not animals received estrogen treatment. Thus, there are sex differences in striatal extracellular dopamine and in the effect of estrogen on the striatal dopamine neurochemical and behavioral responses to amphetamine.
\end{abstract}

\section{INTRODUCTION}

It has been known for some time that there are sex differences in behaviors mediated by the mesostriatal dopamine (DA) system. Female rats in estrus exhibit greater amphetamine (AMPH)-induced rotational behavior than do intact male rats $^{8}$. Females also exhibit more intense stereotyped behaviors in response to AMPH than males ${ }^{1,21}$. Following ovariectomy (OVX) of female rats there is an attenuation of AMPH-induced rotational behavior, electrical stimulation-induced rotational behavior, and AMPH-induced stereotyped behaviors ${ }^{12,18,21}$. During the estrous cycle of the rat, females in estrus exhibit a greater frequency of AMPH-induced stereotypyed behaviors than those in diestrus $^{6}$. Similarly, there is enhanced AMPH-stimulated DA release in stiatum on estrus, compared to other days of the cycle determined either in vitro ${ }^{2,7}$ or in vivo using microdialysis ${ }^{6}$. By contrast, castration (CAST) of male rats has no effect on the behaviors discussed above or on striatal DA release $e^{7,12,18,21}$. These findings suggest that ovarian hormones, but not testicular hormones, modulate mesostriatal DA function in the rat.

Following OVX, AMPH-stimulated DA release from striatal tissue in vitro is attenuated ${ }^{2,7}$. By contrast, following CAST, AMPH-stimulated DA release from striatal tissue in vitro does not diminish ${ }^{7}$. Treatment of OVX rats with estrogen alone or a combination of estrogen and progesterone restores the AMPH-stimulated striatal DA release in vitro ${ }^{4,5,7}$. In vivo, using microdialysis in freely moving rats, a single physiological dose of estrogen administered systemically to OVX rats has been found to enhance AMPH-stimulated striatal DA release ${ }^{3}$. Striatal DA turnover is also enhanced by estrogen treatment ${ }^{13}$. An effect of estrogen on striatal DA function in OVX rats is reflected in enhanced AMPH-induced rotational behavior following estrogen treatment ${ }^{3,4}$. Finally, intrastriatal adminsistration of $17 \beta$-estradiol, but not cholesterol or $17 \alpha$ -

Correspondence: J.B. Becker, The University of Michigan, Neuroscience Laboratory Building, 1103 East Huron St., Ann Arbor, MI 48104-1687, USA. Fax: (1) (313) 936-2690. 
estradiol, significantly enhances the accuracy of foot placement in OVX female rats traversing a suspended narrow beam?.

The present studies were designed to determine whether there are sex differences in the effect of a single acute injection of estrogen on AMPH-stimulated striatal DA release determined by in vivo microdialysis and the associated AMPH-induced behaviors. In addition, possible sex differences in basal extracellular striatal DA concentrations were evaluated using traditional in vivo microdialysis and quantitative in vivo microdialysis. Additional experiments were conducted in an effort to elucidate the possible source of the observed sex-related difference in basal extracellular striatal DA concentrations in dialysate obtained in the initial experiments. We report here for the first time that there are hormone-independent sex differences in the basal extracellular concentration of DA in striatum, and that estrogen enhances AMPH-stimulated striatal DA release and AMPH-induced behaviors in OVX but not CAST rats.

\section{MATERIALS AND METHODS}

\section{Subjects}

Adult male and female Sprague-Dawley rats (Holtzman Co., Madison, WI) were maintained on a 14:10 h LD cycle with food and water available ad libitum.

\section{General experimental procedures}

Surgery. Animals were castrated (CAST) or ovariectomized (OVX) under ether anesthesia using sterile/aseptic conditions. The vaginal epithelium of the OVX females was examined daily for at least ten consecutive days and any animal that continued to have cornified cells in its vaginal epithelium was eliminated from the study.

Microdialysis procedures. At least one month post-gonadectomy, subjects that passed criteria had an $8 \mathrm{~mm}$ guide cannula implanted through the skull on one side of the brain (left/right randomized) under sodium pentobarbitol anesthesia $(30 \mathrm{mg} / \mathrm{kg})$ supplemented with methoxyflurane. Coordinates from bregma (skull flat): anterior $0.5 \mathrm{~mm}$, lateral $2.5 \mathrm{~mm}$, ventral $1 \mathrm{~mm}$. The guide cannula was cemented into place with dental acrylic and a stylet was inserted to keep the cannula patent.

One week post-surgery, subjects were anesthetized with ether and anesthesia was maintained with methoxyflurane. The stylet was removed and a microdialysis probe was lowered through the guide cannula to a depth of $6.5 \mathrm{~mm}$ ventral to skull surface. The dialysis fiber was a semipermeable regenerated cellulose membrane with an outer diameter of $250 \mu \mathrm{m}$ and a molecular weight cutoff of 6000 (Spectrum Medical Corp., VWR Scientific, Chicago, IL). The probe design used has been described in detail by Robinson and Whishaw ${ }^{20}$ Twelve to $16 \mathrm{~h}$ after probe insertion, animals underwent in vivo microdialysis.

During dialysis a Ringer's solution ${ }^{3}$ was pumped at a constant rate of $1.5 \mu \mathrm{l} / \mathrm{min}$ through the probe, unless otherwise noted Concentrations of DA in dialysate were determined by high performance liquid chromatography with coulometric detection ${ }^{3}$. The extent of diffusion for DA and its metabolites, dihydroxyphenyl-acetic acid (DOPAC) and homovanilic acid (HVA), into each probe at $37^{\circ} \mathrm{C}$ was determined prior to use (i.e. \% recovery). Probes were not used if the percent recovery for any of the compounds was outside of the acceptable range $(20-25 \%)$. For the traditional microdialysis experiments, the extracellular concentrations of DA were corrected for the percent recovery determined in vitro. No corrections for recovery in vitro were made to values obtained by the no net flux method, since this method takes into account the rate of DA diffusion into and out of the microdialysis probe (see below).

Histology. Following dialysis, animals were killed with an overdose of sodium pentobarbital and perfused transcardially with $0.9 \%$ saline followed by $10 \%$ formalin. In order to determine probe placement, brains were sectioned at $40 \mu \mathrm{m}$ on a freezing microtome and stained with Cresyl violet. Animals with probe placement outside of the striatum were eliminated from the study.

Statistical analysis. Concentrations of DA in dialysate were corrected for recovery unless otherwise noted and converted to $\mathrm{fmol} / \mu \mathrm{l}$. AMPH-stimulated DA release was also expressed as the increase from basal concentration by subtracting the mean basal DA $(\mathrm{fmol} / \mu \mathrm{l})$ from the stimulated value. Neurochemical and behavioral data were analyzed by analysis of variance (ANOVA) or Student's $t$-tests as indicated. All statistical analyses were performed using Statview $512+$ on a Macintosh Plus.

Effect of estrogen on AMPH-stimulated striatal DA release and AMPH-induced behaviors in CAST and OVX rats

Microdialysis. On the day of dialysis, $6-8$ baseline samples were collected at $15 \mathrm{~min}$ intervals. After baseline samples were collected, animals received injections (s.c.) of estrogen or vehicle as follows: Group 1: estradiol benzoate (EB; $2.0 \mu \mathrm{g} / 100 \mathrm{~g}$ body wt.) or an equivalent volume of vehicle (peanut oil); or Group 2: 17 $\beta$-Estradiol $(1.5 \mu \mathrm{g} / 100 \mathrm{~g})$ or an equivalent volume of vehicle (peanut oil). EB was given to animals in Group 1 in order to replicate previous findings from this laboratory ${ }^{3}$. Since EB must be metabolized to $17 \beta$-estradiol prior to acting in vivo, we also examined the effect of $17 \beta$-estradiol on animals in Group 2 in order to demonstrate that the same results could be obtained with the native compound. The doses were chosen to produce the same physiological concentrations of estradiol in blood during the experiment ${ }^{11,15}$. Following hormone treatment, two additional samples were collected. Thirty minutes after treatment, animals in Group 1 received $2 \mathrm{mg} / \mathrm{kg} \mathrm{D}$-amphetamine sulfate (AMPH, i.p.); animals in Group 2 received $1.25 \mathrm{mg} / \mathrm{kg}$ AMPH (i.p.). A lower dose of AMPH $(1.25 \mathrm{mg} / \mathrm{kg}$ ) was given to animals in Group 2 in order to examine any dose-response relations between the effect of estrogen and dose of AMPH. Following AMPH, eight additional 15 min samples were collected ( 2 hours).

Behavioral measures. Animals in Group 1 were videotaped for the entire two hour period following AMPH. Head/forelimb movements and sniffing were given a rating from $0-4$ based on thirty second samples of behavior taken from the tapes at the start of each 5 min interval post-AMPH. A score of zero indicated that the animal was asleep or inactive, while a score of 4 corresponded to intense stereotyped behavior. In addition, a subset of the tapes were scored quantitatively (a few of the intitial recordings were inadvertantly taped over, so these were unavailable). For these tapes, the number of head and forelimb movements were counted. Sixty second samples of these behaviors were taken twice during each 15 min interval post-AMPH. For animals in Group 2, rotational behavior and general activity (extra quarter turns) were recorded automatically by an automated computer controlled system ${ }^{3}$.

Sex differences in $\left[{ }^{3} \mathrm{H} / D A\right.$ uptake into synaptosomes from striatal tissue

Tissue. Synaptosomal preparation and procedures for $\left[{ }^{3} \mathrm{H}\right] \mathrm{DA}$ uptake were modified from those described by Bonnet et al. ${ }^{10}$. CAST and OVX rats were decapitated and the striatum dissected on ice. Striatal tissue was homogenized in 10 vols. $(w / v)$ of ice cold $0.32 \mathrm{M}$ sucrose. Homogenization was performed by 10 up and down strokes at a pestle revolution of $800 \mathrm{rpm}$. Following homogenization, the pestle and glass vessel were rinsed with an additional 10 vols. of 0.32 $M$ sucrose which was added to the homogenate. The homogenate was centrifuged at $1000 \times \mathrm{g}$ for $10 \mathrm{~min}$ at $4^{\circ} \mathrm{C}$. The supernatant was decanted to yield the crude synaptosomal preparation.

Incubation. Aliquots of the crude synaptosomal preparation $(50 \mu \mathrm{l})$ were added to test tubes containing $450 \mu \mathrm{l}$ of Krebs-Ringer phosphate buffer. The Krebs-Ringer phosphate buffer contained $50 \mu \mathrm{l}$ 
of each of the following compounds: $109 \mathrm{mM} \mathrm{NaCl}, 3.55 \mathrm{mM} \mathrm{KCl}$, $2.4 \mathrm{mM} \mathrm{CaCl}, 0.61 \mathrm{mM} \mathrm{MgSO}, 1.1 \mathrm{mM} \mathrm{NaH} \mathrm{PO}_{4}, 25 \mathrm{mM}$ $\mathrm{KHCO}_{3}, 5.4 \mathrm{mM}$ glucose and $20 \mu \mathrm{M}$ nialimide. Prior to use, the media was bubbled with a gas mixture of $95 \% \mathrm{O}_{2}-5 \% \mathrm{CO}_{2}$ for 30 $\mathrm{min}$ at a $\mathrm{pH}$ of 7.4. After the synaptosomal preparation was added to the tubes containing media, the tubes were incubated for $5 \mathrm{~min}$ in a shaking water bath maintained at $37^{\circ} \mathrm{C}$. Ten $\mu$ lof $\left[{ }^{3} \mathrm{H}\right] \mathrm{DA}\left(2 \times 10^{-8}\right.$ M) was added to each of the tubes (triplicates were run for each incubation time). The tubes were then incubated in a shaking water bath at $37^{\circ} \mathrm{C}$ for $1,2,3,5,10,15$, or $30 \mathrm{~min}$. The reaction was stopped by the addition of $2 \mathrm{ml}$ of ice-cold Ringer's to each tube. All synaptosomal preparations were centrifuged at $7000 \times g$ for $10 \mathrm{~min}$ at $4^{\circ} \mathrm{C}$ and washed with ice-cold Ringer's. This step was performed twice. The remaining pellets were solubilized at $50^{\circ} \mathrm{C}$ for $2-3 \mathrm{~h}$. An aliquot of the above solution was analyzed for $\left[{ }^{3} \mathrm{H}\right] \mathrm{DA}$ uptake using liquid scintillation counting. Protein concentrations in the synaptosomal preparations were determined using the Pierce BCA Protein Assay.

Sex differences in extracellular $D A$ determined by quantitative microdialysis

The technique of quantitative microdialysis was modified from methods described in detail by Parsons and Justice ${ }^{17}$. In these experiments, the Ringer's solution also contained $0,1,4$ or $8 \mathrm{nM}$ DA and was pumped at a constant rate of $1 \mu \mathrm{l} / \mathrm{min}$. The probe design was also modified for these experiments so that the inlet tubing was fused silica instead of PE20 tubing, in order to minimize the volume within the system. On the day of dialysis, 6-8 baseline samples were collected while a Ringer's solution containing $0 \mathrm{nM}$ DA flowed through the probe. After basal DA levels were established, the DA-containing solutions $(1,4$ or $8 \mathrm{nM})$ were perfused through the probe in random order. Eight 20 min samples were collected at each concentration. The last four samples at each concentration were analyzed.

The mean of the four dialysis samples run for each DA concentration was calculated. For each animal, the gain or loss of DA from the brain at each concentration was plotted and a linear regression line drawn through the points. The point at which there was no net gain or loss of DA from the brain was equal to the extracellular DA concentration for that animal (i.e. the 'point of no net flux').

\section{RESULTS}

Effect of estrogen on AMPH-stimulated striatal DA release and AMPH-induced behaviors in CAST and OVX rats

Microdialysis. There was a sex difference in the basal concentration of DA in dialysate from OVX and CAST rats. Data from the baseline period from Groups 1 and

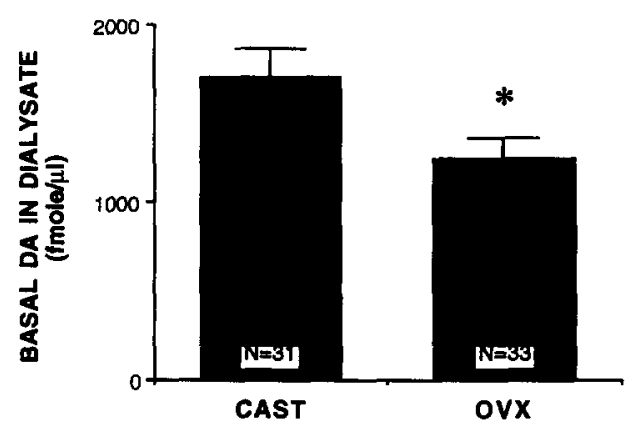

Fig. 1. The mean basal striatal DA concentration in dialysate $\left( \pm\right.$ SEM) for CAST and OVX rats are shown above. ${ }^{*}$ The CAST rats had significantly higher basal striatal $D A$ concentrations than did the OVX rats $\left(t_{62}=2.32, P=0.024\right)$.
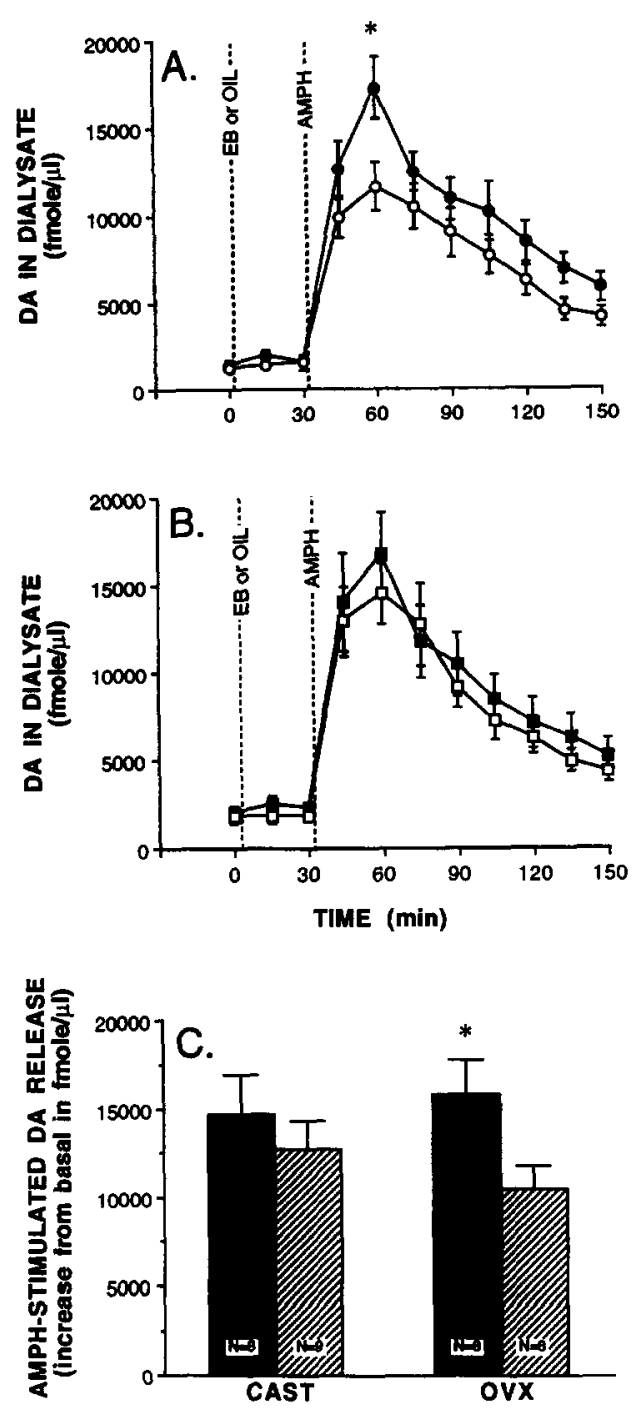

Fig. 2. Group 1: Neurochemistry: A: the time course of AMPHstimulated $(2.0 \mathrm{mg} / \mathrm{kg})$ striatal DA release for the oil-treated OVX rats ( $n=8$; open circles) and the EB-treated OVX rats $(n=8$; closed circles). DA concentrations in dialysate are expressed in $\mathrm{fmol} / \mu \mathrm{l}$ and samples were collected at $15 \mathrm{~min}$ intervals. ${ }^{*}$ The EB-treated females showed a significantly greater peak DA increase from baseline when compared to the oil-treated controls $\left(F_{1,15}=4.598, P=\right.$ 0.05 ). B: there was no significant difference in the timecourse of AMPH-stimulated striatal DA release between the oil-treated CAST rats ( $n=9$; open squares) and the EB-treated CAST group $(n=8$; closed squares) $(P=0.210)$. C: the average $( \pm$ S.E.M. $)$ peak in AMPH-stimulated striatal DA release is expressed as increase from basal in $\mathrm{fmol} / \mu 1$ for CAST and OVX animals in Group 1. * The EB-treated OVX rats (solid bar) showed a significantly greater peak DA increase from basal than did the oil-treated OVX controls (hatched bar) $\left(F_{1,15}=5.507, P=0.034\right)$. There was no difference in the peak DA increase from basal for the CAST groups. All values are the mean \pm S.E.M.

2 plus basal data from animals used in pilot experiments were combined for this analysis. CAST rats had significantly higher basal striatal DA concentrations than OVX rats $\left(t_{62}=2.32, P=0.024\right.$; Fig. 1$)$. The magnitude of the observed sex difference was approximately $27 \%$. 
There was no effect of estrogen on extracellular DA concentrations in the striatum of CAST and OVX rats in either Group 1 or Group 2 during the thirty min collection interval prior to AMPH. In contrast, for both groups there was an effect of estrogen on the AMPH-stimulated increase in striatal DA in dialysate from OVX, but not from CAST rats. As shown in Fig. $2 \mathrm{~A}$, in OVX rats the increase in DA after AMPH was significantly greater for the EB-treated group than the oil-treated controls during the second 15 min collection interval after AMPH $(2.0 \mathrm{mg} / \mathrm{kg} ; P=0.034)$. In contrast, in the CAST groups, EB and oil-treated rats did not differ significantly at any time point after AMPH ( $P=0.210$; Fig. $2 \mathrm{~B})$, including the second interval after AMPH (Fig. 2C).

In Group 2, the $17 \beta$-estradiol-treated OVX rats also showed a significantly greater peak DA increase from baseline 15-30 min after AMPH than the oil-treated controls $(P=0.028$; Fig. 3A). As shown in Fig. $3 \mathrm{~B}$, the $17 \beta$-estradiol-treated CAST rats did not differ significantly from the oil-treated controls at any time point post-AMPH $(P=0.871)$. There was, however, a sex difference. DA release was significantly greater after $1.25 \mathrm{mg} / \mathrm{kg}$ AMPH in striatum of OVX rats than in CAST rats, regardless of hormone treatment (Fig. 3C).

The concentrations of the DA metabolites, DOPAC and HVA, in dialysate did not differ significantly among the four groups for any collection interval, before or after AMPH for either Group 1 or Group 2 (data not shown).

Behavioral measures. For Group 1, there were significant differences between groups in the frequency of head and forelimb movements. To obtain the individual points seen in Fig. 4A, the average of two sixty second samples of behavior taken during a $15 \mathrm{~min}$ interval post-AMPH was calculated. As shown in Figs. $4 \mathrm{~A}$ and $\mathrm{B}$, the EB-treated OVX group made significantly more head and forelimb movements per sixty second sample after AMPH than the oil-treated controls $\left(F_{1,7}=18.933 ; P=0.003\right)$. In contrast, the frequency of head and forelimb movements per sixty second sample for the the EB-treated CAST rats did not differ significantly from the oil-treated CAST group at any time point after $\operatorname{AMPH}\left(F_{1,14}=1.723 ; P=0.210\right.$; Fig. 4A and B). There was also a sex difference, regardless of hormone treatment, in the total number of head and forelimb movements made during the entire two hour period after AMPH (Main effect of sex $-F_{1,23}=13.007 ; P=0.002$; sex $\times$ Time interaction$\left.F_{7,161}=3.174, P=0.004\right)$. During this period, the CAST groups made significantly more stereotyped head and forelimb movements than the OVX groups (Fig. 4B). After $2 \mathrm{mg} / \mathrm{kg}$ AMPH, there were no significant dif- ferences among the four groups in intensity of stereotypy as assessed by a traditional stereotypy rating scale.

For Group 2 there was no significant difference among the four treatment groups in the number of rotations or the total number of $1 / 4$ turns made postAMPH, $(P=0.851$; data not shown $)$. In contrast, there was a sex-related difference in the number of total extra $1 / 4$ turns made after AMPH. Extra $1 / 4$ turns are those $1 / 4$ turns that do not contribute to full turns
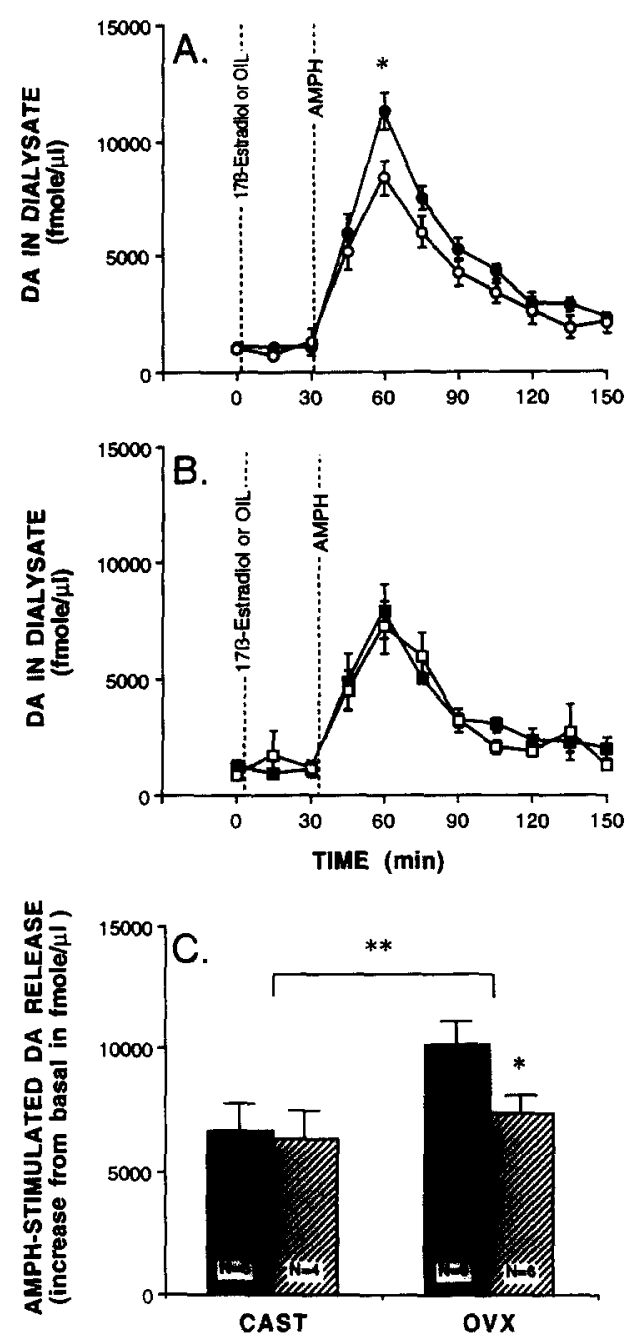

Fig. 3. Group 2: Neurochemistry: A: the time course of AMPHstimulated $(1.25 \mathrm{mg} / \mathrm{kg})$ striatal DA release for the $17 \beta$-estradiol treated OVX rats (closed circles) and the oil-treated OVX controls (open circles) is shown. DA concentrations in dialysate are expressed in $\mathrm{fmol} / \mu \mathrm{l}$ and samples were collected at $15 \mathrm{~min}$ intervals. ${ }^{*}$ The $17 \beta$-estradiol treated females showed a greater peak DA increase from baseline than the oil-treated females $\left(F_{1,11}=5.72, P=0.038\right)$. $B$ : there was no significant difference in AMPH-stimulated striatal DA release across time between the $17 \beta$-estradiol-treated CAST rats ( $n=5$; closed squares) and the oil-treated CAST controls $(n=4$; open squares; $P=0.851) . \mathrm{C}:{ }^{*}$ The $17 \beta$-estradiol-treated females (solid bar) showed a significantly greater peak DA increase $(P=$ 0.028 ) from baseline than did the oil-treated females (hatched bar). In addition, as illustrated by the double asterisks, AMPH-stimulated DA release was significantly higher in the striatum of OVX rats than in striatum of CAST rats regardless of hormone treatment $\left(F_{1,17}=\right.$ $5.538, P=0.031)$. All values are the mean \pm S.E.M. 

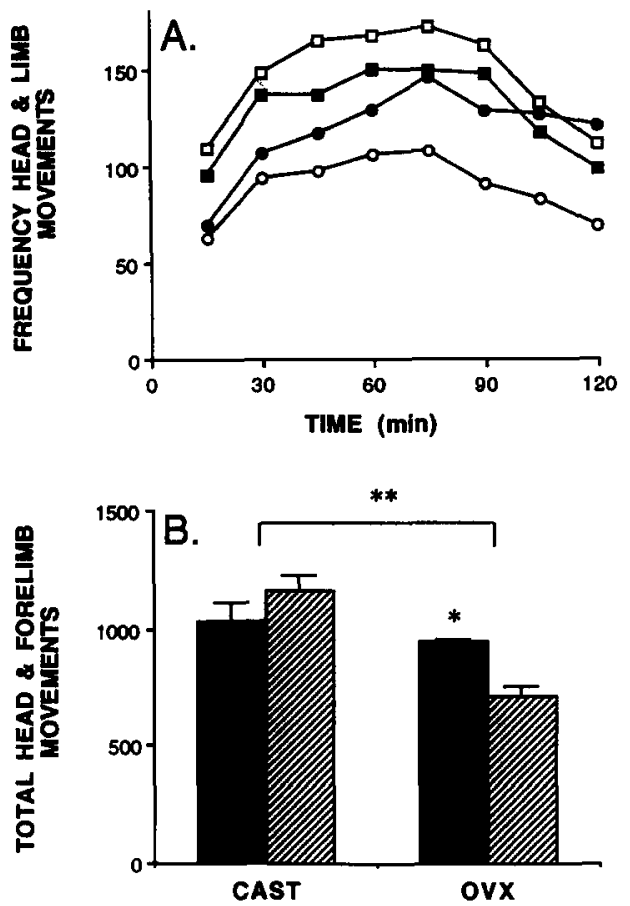

Fig. 4. Group 1: Behavior. A: the average number of head and forelimb movements made per sixty second sample of behavior across time after AMPH $(2.0 \mathrm{mg} / \mathrm{kg})$ for OVX females and CAST males in Group 1. The EB-treated females (closed circles) made a significantly greater number of head and forelimb movements than the oil-treated females (open circles) post-AMPH $\left(F_{1,7}=18.933\right.$, $P=0.003$ ). There was no significant difference in the number of head and forelimb movements made by CAST males after AMPH (EB treated males $=$ closed squares; oil-treated males $=$ open squares; $F_{1,14}=1.723, P=0.210$ ). B: mean \pm S.E.M. of total head and limb movements during the two hour period post-AMPH. * The EB-treated females (solid bar) made a significantly greater number of head and limb movements $(P=0.003)$ during the two hour period post-AMPH than the oil-treated females (hatched bar). ** The CAST rats made significantly more stereotyped head and forelimb movements than the OVX females during the $2 \mathrm{~h}$ following AMPH (main effect of sex $-F_{1,23}=13.007 ; P=0.002$; Sex $\times$ Time interaction $\left.-F_{7,161}=3.174, P=0.004\right)$.

$\left(360^{\circ}\right)$ and are used as an index of general activity ${ }^{14}$. As shown in Fig. 5B, the OVX rats made significantly more extra 1/4 turns than the CAST rats after AMPH, whether or not they received hormone treatment $\left(F_{1,19}\right.$ $=5.599 ; P=0.029$ ). When OVX animals treated with $17 \beta$-estradiol were compared with controls, there was a significant Time $\times$ Hormone treatment interaction $\left(F_{9,90}=2.009 ; P=0.0471\right.$; Fig. 5A), but there was no main effect of hormone treatment $\left(F_{1,10}=0.712, P=\right.$ 0.419). The significant interaction indicates that the peak in extra $1 / 4$ turns is greater in the $17 \beta$-estradiol treated OVX rats than for the OIL treated controls.

Sex differences in $\left[{ }^{3} H\right] D A$ uptake into synaptosomes from striatal tissue

There was no significant difference in striatal DA uptake at any time point. As shown in Fig. 6, the time
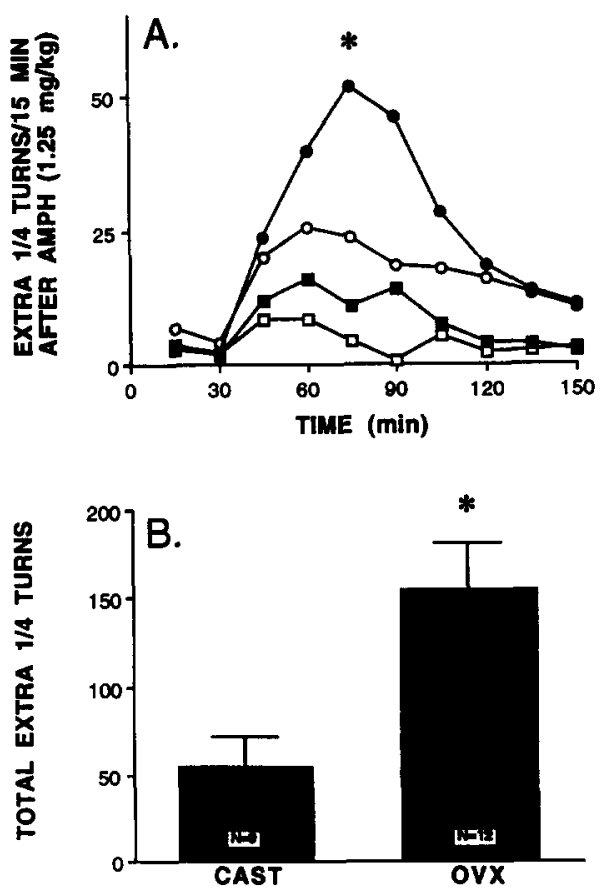

Fig. 5. Group 2: Behavior. A: the mean number of extra 1/4 turns $/ 15 \mathrm{~min}$ after AMPH $(1.25 \mathrm{mg} / \mathrm{kg})$ made by CAST males and OVX females in Group 2. * There was a significant Time $\times$ Hormone treatment interaction when the $17 \beta$-estradiol-treated females (closed circles) were compared to vehicle-treated controls (open circles; $F_{9,90}=2.009, P=0.0471$ ). There was no significant difference between the CAST males (squares) after AMPH. B: mean \pm S.E.M. of extra $1 / 4$ turns per $15 \mathrm{~min}$ during the two hour period post-AMPH. * The OVX females made more extra $1 / 4$ turns than the CAST males during the 2 hour period following AMPH $\left(F_{1,19}=5.599\right.$, $P=0.029$ ).

course of $\left[{ }^{3} \mathrm{H}\right] \mathrm{DA}$ uptake did not differ significantly in synaptosomes from OVX and CAST striatal tissue.

Sex differences in extracellular $D A$ determined by quantitative microdialysis

There was a sex difference, independent of concurrent gonadal hormones, in extracellular striatal DA concentrations assessed by quantitative in vivo micro-

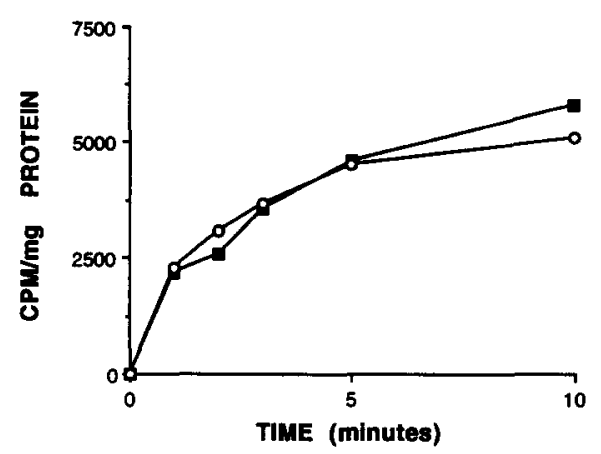

Fig. 6. The timecourse of $\left[{ }^{3} \mathrm{H}\right]$ dopamine uptake by striatal synaptosomes from CAST male rats ( $n=6$; squares) and OVX female rats ( $n=6$; circles) is shown above. Values are expressed in disintegrations per minute/mg protein. Striatal DA uptake did not differ significantly between the two groups. 


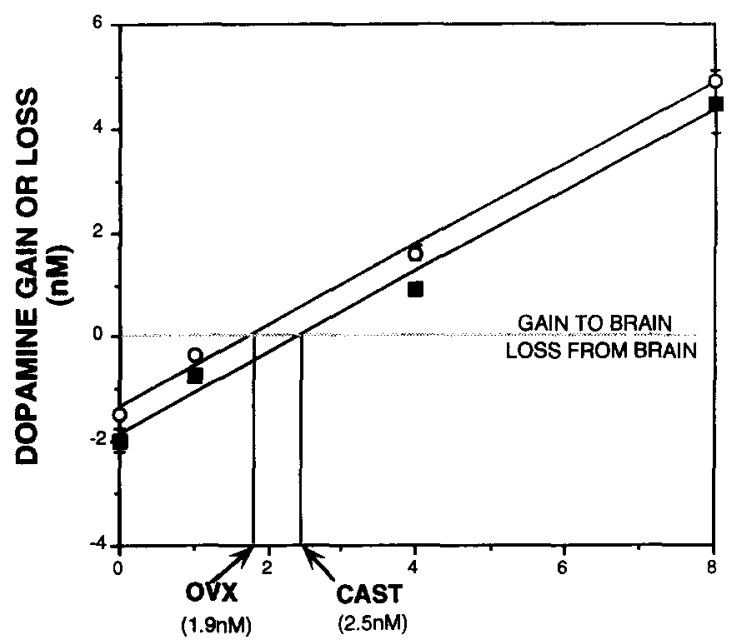

DA ADDED TO PERFUSATE

Fig. 7. For each animal the average gain or loss of DA from the brain at each concentration was plotted and a simple regression line drawn through the points, as illustrated above. The point at which there was no net gain or loss of DA from the probe was equal to the extracellular striatal DA concentration for that animal (i.e. the 'point of no net flux'). The slope of the regression line was equal to the in vivo recovery for that probe. There was no significant difference in the average regression lines for the CAST male rats and the OVX female rats. In contrast, the CAST males had significantly higher extracellular striatal DA concentrations than the OVX female rats $(t=-2.672 ; \mathrm{df}=18 ; P<0.016)$.

dialysis. As can be seen in Fig. 7, CAST rats had significantly higher extracellular striatal DA concentrations than OVX rats $(P<0.016)$. The observed sex difference was approximately 20 percent. There was no difference in the slope of the curve obtained by varying the concentration of DA in the perfusate, indicating that the rate of DA diffusion down its concentration gradient (into the brain or into the probe) was the same for OVX and CAST rats.

\section{DISCUSSION}

The present findings are the first demonstration of a sex difference in basal extracellular striatal DA concentrations determined by quantitative microdialysis that is independent of concurrent gonadal hormones. In addition, $30 \mathrm{~min}$ after a single acute injection of either EB or $17 \beta$-estradiol, AMPH-stimulated striatal DA release was potentiated in OVX rats. The enhanced striatal DA release coincided with an enhanced frequency of stereotyped head and forelimb movements and increased peak in extra 1/4 turns following AMPH treatment in OVX rats. Neither form of estrogen affected AMPH-stimulated striatal DA release or the behavioral response to AMPH of CAST male rats. Taken together these results indicate that estrogen rapidly potentiates AMPH-stimulated striatal DA release and DA-mediated behaviors in the OVX rat.
The observed sex difference in basal striatal DA concentrations might reflect sex differences in one or more neural processes. The sex difference might be due to an actual difference in DA release in striatum of CAST male and OVX female rats. However, this is not the only possibility. A second possibility is that there might be a sex difference in striatal DA uptake. The findings presented in this report, however, indicate that a sex difference in striatal DA uptake is not the source of the sex difference in basal extracellular striatal DA concentrations. Third, the sex difference could be attributable to differences in DA metabolism and/or DA synthesis in the striatum of male and female rats. We found that extracellular concentrations of the DA metabolites DOPAC and HVA were not different in the CAST and OVX groups, so metabolic or synthetic differences are probably not the only factor underlying sex differences in extracellular concentrations of DA in striatum. More direct measurements of DA synthesis and metabolism are needed, however, to be confident on this point. Finally, there could be a sex difference in the tortuosity of striatal tissue. In other words, diffusion of DA through the striatum toward the dialysis probe may be less impeded in male rat striatum than in female rat striatum. According to Parsons and Justice ${ }^{17}$ the slope of the regression line obtained from quantitative dialysis experiments reflects the capability of DA to diffuse across the dialysis membrane in the rat striatum and is related to the tortuosity of the extracellular space. The absence of a sex difference in the slope of the regression lines obtained from the quantitative microdialysis experiments, however, suggests that diffusion factors are not the primary cause of the sex difference reported. Thus, the sex difference in extracellular DA most likely reflects a sex difference in striatal DA release, DA synthesis, and/or metabolism.

The finding that AMPH-stimulated striatal DA release is enhanced in OVX rats, $30 \mathrm{~min}$ after a single injection of either $17 \beta$-estradiol or EB, supports previous results from this laboratory ${ }^{3}$. This is the first demonstration, however, that estrogen treatment does not affect AMPH-stimulated DA release in the striatum of CAST rats in vivo. The effects of estrogen on striatal DA release may be mediated by a direct effect of estrogen on the striatum of the female rat. Administration of estradiol to striatal tissue in vitro enhances $\mathrm{DA}$ release induced by either AMPH or $\mathrm{KCl}$-induced depolarization when tissue is obtained from OVX rats ${ }^{2}$. Since DA release induced by either AMPH or $\mathrm{KCl}$ are both enhanced by estrogen, it has been suggested that estrogen results in hyperpolarization of DA terminals ( see $^{2}$ for discussion). In addition, the effect of estrogen 
on striatal DA release is not a non-specific action of the hormone, since estrogen applied directly to striatal tissue in vitro has no effect on striatal DA release from tissue of male rats ${ }^{2}$ and systemic estrogen treatment in the study reported here has no effect on striatal DA release in CAST rats in vivo. Taken together, these results suggest that estrogen is acting at specific binding sites in the striatum to induce its effects on striatal DA terminals.

The behavioral results obtained support the hypothesis that estrogen modulates AMPH-induced behaviors in OVX rats, but not CAST rats. A single injection of EB rapidly enhanced the frequency of the stereotyped response to AMPH in OVX, but not in CAST rats. These findings support the results obtained in previous studies from this laboratory ${ }^{12,19}$. However, this is the first report of a sex difference in the frequency of AMPH-induced stereotyped head and forelimb movements where CAST rats exhibited a greater frequency of stereotyped head and limb movements than did OVX rats. In most reports, females tend to exhibit either a greater or more intense behavioral response to AMPH than males ${ }^{1,21}$. It is possible that the OVX females were engaging in other, possibly more 'intense', stereotyped behaviors after AMPH than the CAST rats. However, if this was the case, it was not apparent from our behavioral observations. Further studies, utilizing more extensive quantitative analytical methods will need to be conducted in order to verify this sex-related difference. For animals in Group 2, the finding that the OVX groups made significantly more extra $1 / 4$ turns after $1.25 \mathrm{mg} / \mathrm{kg}$ AMPH than the CAST groups supports results from previous studies which reported that handled female rats exhibit greater locomotor behavior or general activity after AMPH than do male rats ${ }^{23}$. The observation that estrogen enhanced AMPH-induced general activity in OVX rats, but not CAST rats, also lends support to the hypothesis that estrogen modulates mesostriatal DA activity and DAmediated behaviors in female rats.

General activity and locomotor activity are usually associated with administration of low doses of AMPH, while stereotypy is produced by higher doses. In fact, stereotyped behavior is by definition coincident with an absence of locomotor activity. Therefore, the finding that OVX rats show greater general activity and less stereotypy after AMPH than CAST males, suggests that OVX rats are less responsive to AMPH than CAST rats. This theoretical framework relating activity and stereotypy seems inconsistant, however, with our finding that estrogen treatment in OVX rats enhanced both AMPH-induced striatal DA release as well as the behavioral response to AMPH. One might have pre- dicted that enhanced DA release after estrogen would decrease general activity and result in enhanced stereotypy, but this was not what was found. Instead, estrogen enhanced both behaviors which suggests that the intensity of activity and stereotypy are independent behaviors, not part of dose-response continuum as generally thought.

In summary, the results of these experiments indicate that the mesostriatal DA system is sexually dimorphic. Extracellular concentrations of DA in the striatum are different in CAST and OVX rats, and there are sex differences in the effects of AMPH and estrogen on striatal DA release and DA-mediated behaviors. Previous research has demonstrated that estrogen can act directly on the striatum to enhance AMPH-induced DA release and sensorimotor function ${ }^{2,9}$. Thus, the effects reported here are likely to be due to sex differences in and differential effects of estrogen on the striatum. The mechanism mediating this effect has not yet been elucidated, but recent research has demonstrated that progesterone metabolites act in the brain at GABA-A binding sites ${ }^{16,22}$. Thus extracellular membrane receptors for steroid hormones have been clearly established. A membrane-associated receptor for estrogen may be a potential mechanism mediating the effects reported here. It seems evident that we are only beginning to fully appreciate the range of effects that steroids can have on brain function and behavior.

Acknowledgements: This research was supported by a grant from the National Science Foundation to J.B.B. (BNS 9021966). S.A.C. was supported by a training grant to the UM Reproductive Sciences Program (HD07048).

\section{REFERENCES}

1 Beatty, W.W. and Holzer, G.A., Sex differences in stereotyped behavior in the rat, Pharmacol. Biochem. Behav., 9 (1978) 777785.

2 Becker, J.B., Direct effect of $17 \beta$-estradiol on striatum: sex differences in dopamine release, Synapse, 5 (1990) 157-164.

3 Becker, J.B., Estrogen rapidly potentiates amphetamine-induced striatal dopamine release and rotational behavior during microdialysis, Neurosci. Lett., 118 (1990) 169-171.

4 Becker, J.B. and Beer, M.E., The influence of estrogen on nigrostriatal dopamine activity: behavioral and neurochemical evidence for both pre- and postsynaptic components, Behav. Brain Res., 19 (1986) 27-33.

5 Becker, J.B., Beer, M.E. and Robinson, T.E., Striatal dopamine release stimulated by amphetamine or potassium: influence of ovarian hormones and the light-dark cycle, Brain Res., 311 (1984) $157-160$

6 Becker, J.B. and Cha, J., Estrous cycle-dependent variation in amphetamine-induced behaviors and striatal dopamine release assessed with microdialysis, Behav. Brain Res., 35 (1989) 117-125.

7 Becker, J.B. and Ramirez, V.D., Sex differences in the amphetamine stimulated release of catecholamines from rat striatal tissue in vitro, Brain Res., 204 (1980) 361-72.

8 Becker, J.B., Robinson, T.E. and Lorenz, K.A., Sex differences and estrous cycle variations in amphetamine-elicited rotational behavior, Eur. J. Pharmacol., 80 (1982) 65-72. 
9 Becker, J.B., Snyder, P.J., Miller, M.M., Westgate, S.A. and Jenuwine, M.J., The influence of estrous cycle and intrastriatal estradiol on sensorimotor performance in the female rat, Pharmacol. Biochem. Behav., 27 (1987) 53-59.

10 Bonnet, J.J., Lemasson, M.H. and Costentin, J., Simultaneous evaluation by a double labelling method of drug-induced uptake inhibition and release of dopamine in synaptosomal preparation of rat striatum, Biochem. Pharmacol., 33 (1984) 2129-2135.

11 Butcher, R.L., Collins, W.E. and Fugo, N.W., Plasma concentration of LH, FSH, prolactin, progesterone and estradiol-17- $\beta$ throughout the 4-day estrous cycle of the rat, Endocrinology, 94 (1974) 1704-1708.

12 Camp, D.M., Becker, J.B. and Robinson, T.E., Sex differences in the effects of gonadectomy on amphetamine-induced rotational behavior in rats, Behav. Neural Biol., 46 (1986) 491-495.

13 Di Paolo, T., Rouillard, C. and Bedard, P., 17\%-Estradiol at a physiological dose acutely increases dopamine turnover in rat brain, Eur. J. Pharmacol., 117 (1985) 197-203.

14 Glick, S. and Ross, D., Right-sided population bias and lateralization of activity in normal rats, Brain Res., 205 (1981) 222-225.

15 Henderson, S.R., Baker, C. and Fink, G., Oestradiol-17 $\beta$ and pituitary responsiveness to luteinizing hormone releasing factor in the rat: a study using rectangular pulses of estradiol-17 $\beta$ monitored by non-chromatographic radioimmunoassay, $J$. Endocrinol., 73 (1977) 441-453.

16 Majewska, M.D., Harrison, N.L., Schwartz, R.D., Barker, J.L. and Paul, S.M., Steroid hormone metabolites are barbiturate-like modulators of the GABA receptor, Science, 232 (1986) 1004-1007.

17 Parsons, L.H. and Justice, J.B., Extracellular concentration and in vivo recovery of dopamine in the nucleus accumbens using microdialysis, J. Neurochem., 58 (1992) 212-218.

18 Robinson, T.E., Camp, D.M. and Becker, J.B., Gonadectomy attenuates turning behavior produced by electrical stimulation of the nigrostriatal dopamine system in female but not male rats, Neurosci. Lett., 23 (1981) 203-208.

19 Robinson, T.E., Camp, D.M., Jacknow, D.S. and Becker, J.B., Sex differences and estrous cycle dependent variation in rotational behavior elicited by electrical stimulation of the mesostriatal dopamine system, Behav. Brain Res., 6 (1982) 273-287.

20 Robinson, T.E. and Whishaw, I.Q., Normalization of extracellular dopamine in striatum following recovery from a partial unilateral 6-OHDA lesion of the substantia nigra: a microdialysis study in freely moving rats, Brain Res., 450 (1988) 209-224.

21 Savageau, M.M. and Beatty, W.W., Gonadectomy and sex differences in the behavioral responses of amphetamine and apomorphine of rats, Pharmacol. Biochem. Behav., 14 (1981) 17-23.

22 Twyman, R.E. and Macdonald, R.L., Neurosteroid regulation of GABA-A receptor single channel kinetic properties, J. Physiol., in press (1992)

23 West, C.H.K. and Michael, R.P., Mild stress influences sex differences in exploratory and amphetamine-enhanced activity in rats, Behav. Brain Res., 30 (1988) 95-97. 